\title{
DETERMINISTIC STATE-CONSTRAINED OPTIMAL CONTROL PROBLEMS WITHOUT CONTROLLABILITY ASSUMPTIONS
}

\author{
Olivier Bokanowski ${ }^{1,2}$, NicOlas Forcadel ${ }^{3}$ AND HASNAA Zidani ${ }^{4}$
}

\begin{abstract}
In the present paper, we consider nonlinear optimal control problems with constraints on the state of the system. We are interested in the characterization of the value function without any controllability assumption. In the unconstrained case, it is possible to derive a characterization of the value function by means of a Hamilton-Jacobi-Bellman (HJB) equation. This equation expresses the behavior of the value function along the trajectories arriving or starting from any position $x$. In the constrained case, when no controllability assumption is made, the HJB equation may have several solutions. Our first result aims to give the precise information that should be added to the HJB equation in order to obtain a characterization of the value function. This result is very general and holds even when the dynamics is not continuous and the state constraints set is not smooth. On the other hand we study also some stability results for relaxed or penalized control problems.
\end{abstract}

Mathematics Subject Classification. 35B37, 49J15, 49Lxx, 49J45, 90C39.

Received August 28, 2009. Revised January 14, 2010 and March 19, 2010.

Published online August 6, 2010.

\section{INTRODUCTION}

This paper deals with the properties of the value function of optimal control problems with state constraints. Consider the following control system:

$$
\dot{y}_{x}(t) \in F\left(y_{x}(t)\right) \text { for a.e. } t>0, \quad y_{x}(0)=x,
$$

where $F: \mathbb{R}^{n} \rightsquigarrow \mathbb{R}^{n}$ is a set-valued map with compact convex nonempty values. Let $\varphi: \mathbb{R}^{n} \longrightarrow \mathbb{R}$ be a given lower semicontinuous function. For a fixed $(x, T) \in \mathbb{R}^{n} \times[0,+\infty)$, consider the following state-constrained control problem:

$$
\vartheta(T, x)=\min \left\{\varphi\left(y_{x}(T)\right), y_{x} \text { is a solution of }(1.1), \text { and } y_{x}(t) \in \mathcal{K} \text { for } t \in[0, T]\right\}
$$

where $\mathcal{K} \subset \mathbb{R}^{n}$ is a closed set of state constraints.

\footnotetext{
Keywords and phrases. Optimal control problem, state constraints, Hamilton-Jacobi equation.

${ }^{1}$ Laboratoire Jacques-Louis Lions, Université Pierre et Marie Curie 75252 Paris Cedex 05, France. boka@math.jussieu.fr

2 UFR de Mathématiques, Site Chevaleret, Université Paris-Diderot, 75205 Paris Cedex, France.

3 CEREMADE, UMR CNRS 7534, Université Paris-Dauphine, Place de Lattre de Tassigny, 75775 Paris Cedex 16, France. forcadel@ceremade.dauphine.fr

${ }^{4}$ Projet Commands, INRIA Saclay \& ENSTA, 32 Bd Victor, 75739 Paris Cedex 15, France. Hasnaa.Zidani@ensta.fr
} 
In the case when $\mathcal{K}=\mathbb{R}^{n}$ and $\varphi$ is continuous, it is known that the value function $\vartheta$ is the unique continuous viscosity solution of a Hamilton-Jacobi equation [3,5]. In presence of state constraints, the continuity of this value function is no longer satisfied, unless the dynamics satisfy a special controllability assumption on the boundary of the state constraints. This assumption called "inward pointing qualification condition (IQ)" was first introduced by Soner in [22]. It asks that at each point of $\mathcal{K}$ there exists a field of the system pointing inward $\mathcal{K}$. Clearly this condition ensures the viability of $\mathcal{K}$ (from any initial condition in $\mathcal{K}$, there exists an admissible trajectory which could stay for ever in $\mathcal{K}$ ). Under this assumption, the value function $\vartheta$ is the unique continuous constrained viscosity solution of a HJB equation (i.e., super solution on $\mathcal{K}$ and subsolution on $\stackrel{\circ}{\mathcal{K}})[13,19-22]:$

where

$$
\partial_{t} \vartheta(t, x)+H\left(x, D_{x} \vartheta(t, x)\right)=0 \quad \text { for } x \in \mathcal{K}, t>0
$$

$$
H(x, p)=\sup _{q \in F(x)}(-q \cdot p) .
$$

Unfortunately, in many control problems, the condition (IQ) is not satisfied and the value function $\vartheta$ could be discontinuous. In this framework, Frankowska introduced in [16-18] another controllability assumption, called "outward pointing condition (OQ)". This assumption states that every point $x$ on the boundary $\partial \mathcal{K}$ can be reached by a trajectory coming from the interior of $\mathcal{K}$. Under this assumption it is still possible to characterize the value function as the unique lower semicontinuous (for short lsc) solution of (1.2).

In absence of any assumption of controllability, the function $\vartheta$ is discontinuous and its characterization becomes more complicate $[4,7,23]$. The main difficulty comes from the fact that the HJB equation (1.2) may admit several solutions (in viscosity sense). However, it is known that the value function is the smallest lsc viscosity supersolution of (1.2). This kind of characterization was used in [14] to propose an algorithm based on viability theory.

Here, we first prove that $\vartheta$ is the unique solution of the Hamilton-Jacobi equation on $\mathcal{K}$ satisfying an additional information at the boundary $\partial \mathcal{K}$. This information can be dropped out whenever a controllability assumption is satisfied, and then the corresponding HJB equation admits a unique viscosity solution. In the case where no controllability assumption is satisfied, the additional information is important to ensure uniqueness. Nevertheless, we will show that it is possible to forget this additional information using relaxation, or penalization, methods. More precisely, if we consider a larger set $\mathcal{K}^{\varepsilon}$ and a solution $u^{\varepsilon}$ of the Hamilton-Jacobi equation on $\mathcal{K}^{\varepsilon}$ (which will not satisfy any additional information on the boundary so that the solution will not be unique), then we prove that $u^{\varepsilon}$ converges to the value function $\vartheta$ as $\varepsilon$ goes to zero. On the other hand, we also investigate the approximation of the value function $\vartheta$ by a sequence of value functions associated to penalized control problems.

The paper is organized as follows. In Section 2, we introduce the problem and give the main results. In this section we precise also the assumptions and fix the notations that will be used in the sequel. The proof of the first main results (that is the characterization of the value function) is given in Section 3. In Section 4, we shall discuss the case when inward or outward qualification assumption holds. Finally, Sections 5 and 6 are devoted to the study of relaxation and penalization methods allowing to approximate the value function.

\section{Main Results}

\subsection{Settings of the problem and preliminary definitions}

Let $F: \mathbb{R}^{n} \rightsquigarrow \mathbb{R}^{n}$ be a set-valued map with closed convex nonempty values, and consider the associated differential inclusion:

$$
\dot{y}_{x}(s) \in F\left(y_{x}(s)\right) \text { for a.e. } s>0, \quad y_{x}(0)=x .
$$

For every given $t>0$ and $x \in \mathbb{R}^{n}$, we denote by $S_{[0, t]}(x)$ the set of absolutely continuous solutions of the system $(2.3)$ defined on $[0, t]$ and starting from $x$ at time $s=0$ :

$$
S_{[0, t]}(x):=\left\{y_{x}, \dot{y}_{x}(s) \in F\left(y_{x}(s)\right) \text { on }[0, t], \text { and } y_{x}(0)=x\right\} .
$$


It is known that under suitable assumptions on $F$ (see (H2)-(H3) below), for any $t>0$ and $x \in \mathbb{R}^{n}$, the set $S_{[0, t]}(x)$ is nonempty. Now, for any subset $\Omega$ of $\mathbb{R}^{n}$, we define the set of admissible trajectories satisfying (2.3) and lying in $\Omega$ by:

$$
S_{[0, t]}^{\Omega}(x):=\left\{y_{x} \in S_{[0, t]}(x), y_{x}(s) \in \Omega \text { for } s \in[0, t]\right\} .
$$

Let $\mathcal{K}$ be a fixed set of $\mathbb{R}^{n}$ and let $\varphi: \mathbb{R}^{n} \longrightarrow \mathbb{R}$ be a $n$ function satisfying:

(H1) $\varphi$ is a bounded lower semicontinuous function (lsc). Let $M_{0}>0$ such that $|\varphi(x)| \leq M_{0}$, for every $x \in \mathcal{K}$. We can assume, without loss of generality, that $M_{0}<1$ and $\varphi \equiv 1$ on $\mathcal{K}^{c}$.

We stress on that the assumption $M_{0}<1$ is not restrictive. The value 1 is just used as a reference constant and can be replaced by any other constant.

For a fixed $(x, t) \in \mathbb{R}^{n} \times[0,+\infty)$, consider the following state-constrained control problem:

$$
\min \left\{\varphi\left(y_{x}(t)\right), y_{x} \in S_{[0, t]}^{\mathcal{K}}(x)\right\}
$$

The value function $\vartheta: \mathbb{R}^{+} \times \mathbb{R}^{n} \longrightarrow \mathbb{R}$ associated to this problem is defined by:

$$
\vartheta(t, x):= \begin{cases}\min \left\{\varphi\left(y_{x}(t)\right), y_{x} \in S_{[0, t]}^{\mathcal{K}}(x)\right\} & \text { if } S_{[0, t]}^{\mathcal{K}}(x) \neq \emptyset \\ 1 & \text { otherwise. }\end{cases}
$$

Remark 2.1. Let us remark that we have adopted the convention $\vartheta(x, t)=1$, when the set of admissible trajectories is empty: $\mathcal{S}_{[0, t]}^{\mathcal{K}}(x)=\emptyset$. Of course, we can change the value 1 by any other constant bigger than $\|\varphi\|_{L^{\infty}(\mathcal{K})}$, and possibly equal to $+\infty$. The only advantage in taking a finite constant is to have to deal with finite valued functions. This is also useful for numerical purpose.

In the sequel, we assume the following assumptions:

(H2) The set-valued map $F$ is upper semicontinuous (usc) and has nonempty compact convex images.

(H3) There exists $k>0$ such that for every $x \in \mathbb{R}^{n}$, we have $\sup _{v \in F(x)}\|v\| \leq k(1+\|x\|)$.

(H4) $\mathcal{K}$ is a nonempty closed subset of $\mathbb{R}^{n}$.

For some results, we will also assume more regularity on $F$ :

(H5) For every $R>0, \exists C_{R}>0$ such that $F$ is $C_{R}$-Lipschitz on the ball $B(0, R)$ centred on 0 and with radius $R$.

Remark 2.2. We know that under assumptions (H2)-(H3), for every $x \in \mathbb{R}^{n}$ and every $T>0$, the differential equation (2.3) admits an absolutely continuous solution $y_{x}$ defined on $[0, T]$ (see [1]) and that the sets $S_{[0, T]}(x)$ and $S_{[0, T]}^{\mathcal{K}}(x)$ are compact. Moreover, under assumptions (H1)-(H4), the value function $\vartheta$ is lower semicontinuous.

Let us recall that under assumptions (H2)-(H3) there also exists for all negative times a backward trajectory of the system with the initial point $x$, that is an absolutely continuous solution of:

$$
\dot{y}(s) \in F(y(s)) \text { for a.e. } s<0, \quad y(0)=x .
$$

We keep the notation $y_{x}$ for a solution of (2.6). Now, for every subset $\Omega$ of $\mathbb{R}^{n}$ and for each $x \in \Omega$, we define the set $S^{\Omega,-}(x)$ of backward trajectories solutions of $(2.6)$, lying in $\Omega$ during an interval $[-\nu, 0]$, for some $\nu>0$, and arriving at $x$ :

$$
S^{\Omega,-}(x)=\left\{y_{x} \text { solution of }(2.6), \exists \nu>0, y_{x}(s) \in \Omega \text { for } s \in[-\nu, 0]\right\}
$$

Of course, for any $x$ in $\stackrel{\circ}{\Omega}$, the set $S^{\Omega,-}(x)$ is non empty, while for $x \in \partial \Omega$ this set can be empty if all the trajectories arriving at $x$ come from outside $\Omega$.

We now introduce some definitions which will be useful to characterize $\vartheta$. 
Definition 2.3 (contingent epiderivative and $F$-derivative). Consider a function $\phi: \mathbb{R}^{+} \times \mathbb{R}^{n} \longrightarrow \mathbb{R}^{\text {. }}$

(i) The contingent epiderivative of $\phi$ at $(t, x) \in \mathbb{R}^{+} \times \mathbb{R}^{n}$ in the direction $(\tau, z) \in \mathbb{R} \times \mathbb{R}^{n}$ is defined by

$$
D_{\uparrow} \phi(t, x)(\tau, z)=\liminf _{\substack{h \rightarrow 0^{+} \\ z^{\prime} \rightarrow z s \rightarrow \tau}} \frac{\phi\left(t+h s, x+h z^{\prime}\right)-\phi(t, x)}{h} .
$$

(ii) Let $(t, x) \in \mathbb{R}^{+} \times \mathbb{R}^{n}$ and let $y$ be a trajectory solution of (2.6) and arriving at $x$ at time $t=0$. The $F$-derivative of $\phi$ at $(t, x)$ in the direction of $y$ is defined by:

$$
D_{\uparrow}^{y} \phi(t, x)=\liminf _{h \rightarrow 0^{+}} \frac{\phi(t+h, y(-h))-\phi(t, x)}{h} .
$$

(iii) The subdifferential of $\phi$ at point $(t, x)$, denoted $D^{-} \phi(t, x)$, is the set of all vectors $\left(q_{t}, q_{x}\right) \in \mathbb{R} \times \mathbb{R}^{n}$ satisfying:

$$
\phi(s, z) \geq \phi(t, x)+q_{t}(s-t)+\left\langle q_{x}, z-x\right\rangle+o(|s-t|+|z-x|)
$$

as $\mathbb{R}^{+} \times \mathbb{R}^{n} \ni(s, z) \longrightarrow(t, x)$.

The contingent epiderivative is introduced in [1,2]. This notion is used in [16-18] to define the viscosity solution for HJB equations. Here we have introduced also the $F$-derivative at a point $(t, x)$. This derivative gives information on the variation of a function $\phi$ along a trajectory arriving at $x$ at time $t$.

From [2], Chapter 6, we have the following result:

$$
\left(q_{t}, q_{x}\right) \in D^{-} \phi(t, x) \Longleftrightarrow q_{t} \tau+\left\langle q_{x}, z\right\rangle \leq D_{\uparrow} \phi(t, x)(\tau, z) \quad \forall \tau \in \mathbb{R}, z \in \mathbb{R}^{n} .
$$

\subsection{Main results}

Consider the Hamiltonian function $H: \mathbb{R}^{n} \times \mathbb{R}^{n} \longmapsto \mathbb{R}$ defined by

$$
H(x, q):=\sup _{p \in F(x)}\langle-p, q\rangle
$$

We now state the first main result:

Theorem 2.4 (characterisation of the value function). Let $u: \mathbb{R}^{n} \times \mathbb{R} \mapsto\left[-M_{0}, M_{0}\right] \cup\{1\}$ be a lsc function. We assume that $(\mathrm{H} 1)-(\mathrm{H} 4)$ hold. the following assertions are equivalent:

(i) $u=\vartheta$ on $\mathcal{K}$.

(ii) $u$ satisfies

$$
\begin{aligned}
& \forall t>0, x \in \mathcal{K}, \sup _{p \in F(x)}-D_{\uparrow} u(t, x)(-1, p) \geq 0, \\
& \forall t \geq 0, x \in \mathcal{K}, \quad \sup _{y \in S^{\mathcal{K}},-(x)} D_{\uparrow}^{y} u(t, x) \leq 0, \\
& \forall x \in \mathcal{K}, \quad u(0, x)=\varphi(x), \quad \text { and } \quad \forall t>0, x \in \mathcal{K}^{c}, \quad u(t, x)=1,
\end{aligned}
$$

where $\sup _{y \in S^{\mathcal{K},-}(x)} D_{\uparrow}^{y} u(t, x):=-\infty$ when $S^{\mathcal{K},-}(x)=\emptyset$.

If we assume moreover that (H5) holds true, then (i) and (ii) are also equivalent to the following assertion:

(iii) $u$ is a bilateral viscosity solution (see Def. 2.6 below) of

$$
\begin{array}{ll}
\partial_{t} u(t, x)+H\left(x, D_{x} u(t, x)\right) \geq 0 & \forall t>0, x \in \mathcal{K}, \\
\partial_{t} u(t, x)+H\left(x, D_{x} u(t, x)\right)=0 & \forall t>0, x \in \stackrel{\circ}{\mathcal{K}}
\end{array}
$$


and $u$ satisfies the boundary conditions:

$$
\begin{aligned}
& \forall x \in \mathcal{K}, \quad \liminf _{t \rightarrow 0^{+}, y \rightarrow x} u(t, y)=\varphi(x), \\
& \forall x \in \mathcal{K}, \quad u(0, x)=\varphi(x) \quad \text { and } \quad \forall t>0, \forall x \in \mathcal{K}^{c}, u(t, x)=1, \\
& \forall t \geq 0, \forall x \in \partial \mathcal{K}, \sup _{y \in S_{\mathcal{K},-}(x)} D_{\uparrow}^{y} u(t, x) \leq 0 .
\end{aligned}
$$

Remark 2.5. Let us point out that condition (2.8a) is equivalent to the classical notion of superoptimality of the value function (which means that this function is nonincreasing along at least one trajectory satisfying the differential inclusion (2.3)). The sketch of the proof of this known result will be given in Section 3.1.

Condition (2.8b) is less standard, it translates a notion of weak increase of the value function along admissible backward trajectories (satisfying (2.3) and lying in $\mathcal{K}$ ). When $\mathcal{K}$ is equal to $\mathbb{R}^{n}$, or when $\mathcal{K}$ is an open set, we can prove that condition $(2.8 \mathrm{~b})$ can be expressed, in an infinitesimal way, by using the classical notion of epi-derivative (see Prop. 3.5).

Definition 2.6 (bilateral viscosity solution of (2.9)). Let $u: \mathbb{R}_{+} \times \mathbb{R}^{n} \rightarrow\left[-M_{0}, M_{0}\right] \cup\{1\}$ be a lsc function. We say that $u$ is a bilateral viscosity solution of (2.9) iff:

(i) $u$ is a bilateral solution of $(2.9 \mathrm{~b})$ in $\stackrel{\circ}{\mathcal{K}}$, that is, for every $(t, x) \in \mathbb{R}_{*}^{+} \times \stackrel{\circ}{\mathcal{K}}$ and every $\left(q_{t}, q_{x}\right) \in D^{-} u(t, x)$, we have:

$$
q_{t}+H\left(x, q_{x}\right)=0 .
$$

(ii) $u$ satisfies $(2.9 \mathrm{a})$ on the boundary in the sense that for every $(t, x) \in \mathbb{R}_{*}^{+} \times \partial \mathcal{K}$ and every $\left(q_{t}, q_{x}\right) \in$ $D^{-} u(t, x)$, we have:

$$
q_{t}+H\left(x, q_{x}\right) \geq 0
$$

(iii) $u$ satisfies $(2.9 \mathrm{c})-(2.9 \mathrm{e})$ pointwise.

Remark 2.7. The bilateral viscosity notion could be also defined by using the "touching from one side" test functions, see $[3,16]$.

In the case when $F$ is continuous, the characterization given in the above theorem amounts to saying that the value function is the only lsc solution of the HJB equation (2.9a)-(2.9b), satisfying the boundary condition $(2.9 \mathrm{c})-(2.9 \mathrm{~d})$ and the additional information $(2.9 \mathrm{e})$ with respect to any trajectory arriving at $x \in \partial \mathcal{K}$ and lying in $\mathcal{K}$ during an interval $[-\nu, 0]$, for some $\nu>0$. This general characterization, and mainly information $(2.9 \mathrm{e})$, seems difficult to handle in a numerical approximation algorithm for $\vartheta$. However this theoretical result explains clearly why the value function $\vartheta$ cannot be characterized only by means of a classical HJB equation. Actually, equation $(2.9 \mathrm{a})-(2.9 \mathrm{~b})$ does not contain the information that $\vartheta\left(\cdot, y_{x}(\cdot)\right)$ is decreasing, for any $x \in \partial \mathcal{K}$, along the admissible trajectories $y_{x}(\cdot) \in \mathcal{S}^{\mathcal{K},-}(x)$ arriving at $x$. We shall see in Section 4 , that either under inward-pointing or outward-pointing controllability assumption, assertion (2.9e) is satisfied whenever $u$ is a solution of the constrained HJB equation (2.9a)-(2.9b). However, in the general case when no controllability assumption is made, the equation (2.9a)-(2.9b) with boundary conditions $(2.9 \mathrm{c})-(2.9 \mathrm{~d})$ may have several lsc solutions. Among these solutions, the value function is the only one which satisfies also the property (2.9e).

In the sequel, we want to show how it is possible to approximate the value function by a sequence of solutions of HJB equations. The first approach consists in enlarging the set $\mathcal{K}$. More precisely, for every $\varepsilon>0$, let $\mathcal{K}_{\varepsilon}$ be a closed subset of $\mathbb{R}^{n}$ defined by:

$$
\mathcal{K}_{\varepsilon}:=\left\{x \in \mathbb{R}^{n} ; d(x, \mathcal{K}) \leq \varepsilon\right\},
$$

where $d(\cdot, \mathcal{K})$ denotes the distance function to the set $\mathcal{K}$. Then, we have the following result: 
Theorem 2.8 (relaxation method). Assume (H1)-(H5).

For every $\varepsilon>0$, consider $u_{\varepsilon}: \mathbb{R}^{+} \times \mathbb{R}^{n} \rightarrow\left[-M_{0}, M_{0}\right] \cup\{1\}$ a lsc solution of the equation

$$
\begin{aligned}
& \partial_{t} u(t, x)+H\left(x, D_{x} u(t, x)\right) \geq 0 \quad \forall t>0, x \in \mathcal{K}_{\varepsilon}, \\
& \partial_{t} u(t, x)+H\left(x, D_{x} u(t, x)\right)=0 \quad \forall t>0, x \in \stackrel{\circ}{\mathcal{K}}_{\varepsilon}, \\
& \forall x \in \stackrel{\circ}{\mathcal{K}}_{\varepsilon}, \quad \liminf _{t \rightarrow 0^{+}, y \rightarrow x} u(t, y)=\varphi(x)=u(0, x), \\
& \forall x \in \mathcal{K}_{\varepsilon}, \quad u(0, x)=\varphi(x) \quad \text { and } \quad \forall t>0, x \in \mathcal{K}_{\varepsilon}^{c}, u(t, x)=1 .
\end{aligned}
$$

Then, for every $x \in \mathbb{R}^{n}$ and every $t>0$, we have:

$$
\lim _{\varepsilon \rightarrow 0} u_{\varepsilon}(t, x)=\vartheta(t, x)
$$

Let us emphasize that enlarging the set $\mathcal{K}$ is not for ensuring uniqueness of the solution of (2.10). The above result says that we can take, for every $\varepsilon>0$, any lsc solution of (2.10), and then the convergence result (2.11) holds.

Equation (2.10) can also be rewritten as a HJB equation with a Dirichlet boundary condition:

Proposition 2.9 (Dirichlet formulation of the HJB equation). Assume (H1)-(H5). Let $\varepsilon \geq 0$ be fixed and let $u$ be a lsc function on $\mathbb{R}^{+} \times \mathbb{R}^{n}$ with $u(t,)=$.1 on $\mathcal{K}^{c}$. Then the two following statements are equivalent:

(i) $u$ is lsc viscosity solution of (2.10).

(ii) $u$ is lsc viscosity solution (see Def. 2.10) of the Dirichlet problem:

$$
\begin{aligned}
& \partial_{t} u(t, x)+H\left(x, D_{x} u(t, x)\right)=0 \quad x \in \stackrel{\circ}{\mathcal{K}}_{\varepsilon}, t>0, \\
& u(t, x)=1 \quad x \in \partial \mathcal{K}_{\varepsilon}, t>0, \\
& \forall x \in \mathcal{K}_{\varepsilon}, u(0, x)=\varphi(x), \quad \text { and } \quad \forall x \in \stackrel{\circ}{\mathcal{K}}_{\varepsilon}, \liminf _{\substack{t \rightarrow 0+\\
z \rightarrow x}} u(t, z)=u(0, x) .
\end{aligned}
$$

Definition 2.10 (bilateral viscosity solution of (2.12)). Let $u: \mathbb{R}_{+} \times \mathbb{R}^{n} \rightarrow\left[-M_{0}, M_{0}\right] \cup\{1\}$ be a lower semicontinuous function and let $\Omega$ be a closed subset of $\mathbb{R}^{n}$. We say that $u$ is a lsc viscosity solution on $\Omega$ of

$$
\begin{aligned}
& \partial_{t} u(t, x)+H\left(x, D_{x} u(t, x)\right)=0 \quad x \in \stackrel{\circ}{\Omega}, t>0, \\
& u(t, x)=1 \quad x \in \partial \Omega, t>0, \\
& \forall x \in \Omega, u(0, x)=\varphi(x) \text { and } \forall x \in \stackrel{\circ}{\Omega}, \liminf _{\substack{t \rightarrow 0^{+} \\
z \rightarrow x}} u(t, z)=u(0, x),
\end{aligned}
$$

iff

(i) $u$ is a bilateral solution of $(2.13 \mathrm{a})$ in $\stackrel{\circ}{\Omega}$, that is, for every $(t, x) \in \mathbb{R}_{+}^{*} \times \stackrel{\circ}{\Omega}$ and every $\left(q_{t}, q_{x}\right) \in D^{-} u(t, x)$, we have:

$$
q_{t}+H\left(x, q_{x}\right)=0 .
$$

(ii) $u$ satisfies the boundary condition $(2.13 \mathrm{~b})$ in the sense that for every $(t, x) \in \mathbb{R}_{+}^{*} \times \partial \Omega$ and every $\left(q_{t}, q_{x}\right) \in D^{-} u(t, x)$, we have:

$$
\max \left(q_{t}+H\left(x, q_{x}\right), u-1\right) \geq 0 \text {. }
$$

(iii) $u$ satisfies the initial condition $(2.13 \mathrm{c})$ pointwise. 
Remark 2.11. First order HJB equations with boundary conditions arise usually in optimal exit time problems. These problems have been studied in several papers [6-8,11,13]. It is known, that for HJB equations with Dirichlet boundary conditions, uniqueness results for viscosity solution do not hold when no controllability assumption is made on the boundary of the domain to exit.

Now, consider, for every $\varepsilon>0$, the continuous function given by:

$$
g_{\varepsilon}(x)=\min \left(-1+\frac{1}{\varepsilon} d(x, \mathcal{K}), 1\right) \text { for } x \in \mathbb{R}^{n} .
$$

Then we have:

Theorem 2.12 (penalization method). Assume that (H1)-(H5) hold true.

For every $\varepsilon>0$, consider $\vartheta_{\varepsilon}$ the unique lsc bounded viscosity solution of

$$
\begin{aligned}
& \min \left(\partial_{t} v(t, x)+H\left(x, D_{x} v(t, x)\right), v(t, x)-g_{\varepsilon}(x)\right)=0 \quad x \in \mathbb{R}^{n}, t>0, \\
& v(0, x)=\max \left(\varphi(x), g_{\varepsilon}(x)\right) \quad x \in \mathbb{R}^{n} .
\end{aligned}
$$

Then, for every $x \in \mathcal{K}$ and every $t>0$, we have:

$$
\lim _{\varepsilon \searrow 0^{+}} \vartheta_{\varepsilon}(t, x)=\vartheta(t, x)
$$

Remark 2.13. In the above theorem, the value function $\vartheta$ is characterized as the limit of a sequence of (unique) viscosity solutions of variational inequalities. For every $\varepsilon>0$, we will prove that $\vartheta_{\varepsilon}$ is the value function of a penalized control problem with a particular $L^{\infty}$-norm penalization, see Section 6 .

Remark 2.14. In this paper, in order to simplify the presentation of the main results and main ideas, we have focused on Mayer's problem. Yet, with a simple change of variables it is easy to extend all the results to the general Bolza problem (i.e., when the cost function includes also a distributive part).

\section{Proof of Theorem 2.4}

\subsection{Preliminary results}

Firstly, we give some properties satisfied by the value function $\vartheta$.

Proposition 3.1 (dynamic programming principle). Assume (H1)-(H4). Then the value function $\vartheta$ satisfies the following:

(i) For all $x \in \stackrel{\circ}{\mathcal{K}}$,

$$
\liminf _{t \rightarrow 0^{+}, z \rightarrow x} \vartheta(t, z)=\vartheta(0, x)=\varphi(x) .
$$

(ii) Dynamic programming principle: For all $x \in \mathbb{R}^{n}$, for all $t>0$ and $\tau \in[0, t]$, we have:

$$
\left\{\begin{array}{lll}
\vartheta(t, x)=\min _{y_{x} \in S_{[0, \tau]}^{\mathcal{K}}(x)} \vartheta\left(t-\tau, y_{x}(\tau)\right) & & \text { if } S_{[0, \tau]}^{\mathcal{K}}(x) \neq \emptyset, \\
\vartheta(t, x)=1 & & \text { otherwise. }
\end{array}\right.
$$

(iii) Backward dynamic programming principle: For all $x \in \mathbb{R}^{n}, t \geq 0, y_{x} \in S^{\mathcal{K},-}(x)$, and $\tau$ small enough, we have:

$$
\vartheta(t, x) \geq \vartheta\left(t+\tau, y_{x}(-\tau)\right) .
$$


Proof. (i) By definition of $\vartheta$, we know that $\vartheta(0, x)=\varphi(x)$ for every $x \in \mathcal{K}$. Now, let $x$ be in $\stackrel{\circ}{\mathcal{K}}$ and consider two sequences $\left(z_{i}\right)_{i \geq 1} \subset \stackrel{\circ}{\mathcal{K}}$ and $\left.\left(t_{i}\right)_{i} \subset\right] 0,+\infty[$ such that

$$
t_{i} \longrightarrow 0, \quad z_{i} \rightarrow x, \quad \text { and } \vartheta\left(t_{i}, z_{i}\right) \longrightarrow \liminf _{\substack{t \rightarrow 0^{+} \\ z \rightarrow x}} \vartheta(t, z) \quad \text { when } i \rightarrow+\infty
$$

For every $i$, we consider a trajectory $y_{i}$ solution of: $\dot{y}_{i}(s) \in F\left(y_{i}(s)\right)$ on $\left(0, t_{i}\right)$ and $y\left(t_{i}\right)=x$. Since $x \in \stackrel{\circ}{\mathcal{K}}$, for $i$ big enough, $y_{i}(0) \in \stackrel{\circ}{\mathcal{K}}$ and $y_{i}$ belongs to $\mathcal{S}_{\left[0, t_{i}\right]}^{\mathcal{K}}\left(y_{i}(0)\right)$. Hence

$$
\vartheta\left(t_{i}, y_{i}(0)\right) \leq \varphi\left(y_{i}\left(t_{i}\right)\right)=\varphi(x)
$$

Using the fact that $y_{i}(0) \rightarrow x$ at $i \rightarrow \infty$, it yields

$$
\liminf _{\substack{t \rightarrow 0+\\ z \rightarrow x}} \vartheta(t, z) \leq \lim _{i \rightarrow+\infty} \vartheta\left(t_{i}, y_{i}(0)\right) \leq \varphi(x)
$$

Taking into account the fact that $\vartheta$ is lsc, we conclude (i).

The dynamic programming principle (ii)-(iii) can be easily obtained from the definition of $\vartheta$.

In the sequel of the section, we give some interpretations to the sub and superoptimality principles of the DPP. These statements are well known in the case where the state is not constrained (i.e. $\mathcal{K}=\mathbb{R}^{n}$ ) $[3,16]$. In presence of state constraints, our statements can be seen as a generalization of those studied in $[17,18]$ where a qualification condition is required.

Proposition 3.2 (super-optimality). Let $u: \mathbb{R}^{+} \times \mathbb{R}^{n} \longrightarrow \mathbb{R}$ be a bounded lower semicontinuous function. Assume (H2)-(H3), then the following three statements are equivalent:

(i) $\forall t>0, \forall x \in \mathbb{R}^{n}$, we have:

$$
\sup _{p \in F(x)}-D_{\uparrow} u(t, x)(-1, p) \geq 0
$$

(ii) $\forall(t, x) \in \mathbb{R}^{+} \times \mathbb{R}^{n}, \exists y_{x} \in S_{[0, t]}(x), \forall \tau \in[0, t], u\left(t-\tau, y_{x}(\tau)\right) \leq u(t, x)$.

(iii) $\forall t>0, \forall x \in \mathbb{R}^{n}$ and $\forall\left(q_{t}, q_{x}\right) \in D^{-} u(t, x)$, we have:

$$
q_{t}+H\left(x, q_{x}\right) \geq 0 \text {. }
$$

Proof. This proposition is a straightforward consequence of [16], Theorem 3.2 and Lemma 4.3, using the change of variable $\tau \rightarrow t-\tau$.

Remark 3.3. Let us point out that assertion (ii) of the above proposition insures the existence of a trajectory $y_{x}$, starting from $y_{x}(0)=x$, and such that $u\left(t-\cdot, y_{x}(\cdot)\right)$ is decreasing. Nothing guarantees that $y_{x}$ is admissible (and stays in $\mathcal{K}$ ). In the case when the trajectory $y_{x}$ leaves $\mathcal{K}$, if we assume further that $u$ is bounded from above by 1 and $u(s, z)=1$ whenever $z \notin \mathcal{K}$, then we obtain $u\left(t-\cdot, y_{x}(\cdot)\right) \equiv 1$ on $[0, t]$.

An immediate consequence of the above proposition is the following:

Corollary 3.4 (super-optimality of the value function). Assume (H1)-(H4). Then, the value function $\vartheta$ satisfies for all $t>0, x \in \mathbb{R}^{n}$

$$
\sup _{p \in F(x)}-D_{\uparrow} \vartheta(t, x)(-1, p) \geq 0
$$


Proof. Let $(t, x)$ be in $\mathbb{R}^{+} \times \mathbb{R}^{n}$. We claim that

$$
\exists y_{x} \in S_{[0, t]}(x), \forall \tau \in[0, t], \quad \vartheta\left(t-\tau, y_{x}(\tau)\right) \leq \vartheta(t, x)
$$

To prove this assertion, we first consider the case when $S_{[0, t]}^{\mathcal{K}}(x) \neq \emptyset$. By compactness of $S_{[0, t]}^{\mathcal{K}}(x)$ and since $\vartheta$ is lsc, we obtain from Proposition 3.1(ii) the existence of $y_{x} \in S_{[0, t]}^{\mathcal{K}}(x) \subset S_{[0, t]}(x)$ such that $\vartheta\left(0, y_{x}(t)\right)=\vartheta(t, x)$. Hence, for all $\tau \in[0, t]$, we get from Proposition 3.1(ii) (with $z=y_{x}(\tau)$ ) that

$$
\vartheta(t-\tau, z) \leq \vartheta\left(0, y_{z}(t-\tau)\right) \leq \vartheta\left(0, y_{x}(t)\right) \leq \vartheta(t, x)
$$

Now, if $S_{[0, t]}^{\mathcal{K}}(x)=\emptyset$, thus the definition of $\vartheta$ yields $\vartheta(t, x)=1$. Moreover, since $\vartheta$ is bounded by 1 , for any trajectory $y_{x} \in S_{[0, t]}(x)$, we have

$$
\vartheta\left(t-\tau, y_{x}(\tau)\right) \leq 1=\vartheta(t, x)
$$

Therefore, (3.19) is always satisfied for any $(t, x) \in \mathbb{R}^{+} \times \mathbb{R}^{n}$. We conclude by Proposition 3.2.

Proposition 3.5 (sub-optimality). Assume $(\mathrm{H} 2)-(\mathrm{H} 3)$ and let $u: \mathbb{R}^{+} \times \mathbb{R}^{n} \longrightarrow \mathbb{R}$ be a lower semicontinuous function and $\Omega$ be a subset of $\mathbb{R}^{n}$. Then the following two statements are equivalent:

(i) $\forall t \geq 0$ and $\forall x \in \Omega, \sup _{y \in S^{\Omega,-}(x)} D_{\uparrow}^{y} u(t, x) \leq 0$.

(ii) $\forall t \geq 0, \forall x \in \Omega, \forall \tau \in[0, t]$ and $\forall y_{x} \in S_{[0, \tau]}^{\Omega}(x)$, we have $u(t, x) \leq u\left(t-\tau, y_{x}(\tau)\right)$.

Furthermore, if (H5) is also satisfied and $\Omega$ is an open subset, then (i) and (ii) are equivalent to each of the following assertions:

(iii) $\forall t \geq 0, \forall x \in \Omega, \max _{p \in F(x)} D_{\uparrow} u(t, x)(1,-p) \leq 0$.

(iv) (a) $\forall x \in \Omega, \liminf _{t \rightarrow 0, \Omega \ni y \rightarrow x} u(t, y)=u(0, x)$.

(b) $\forall t>0, \forall x \in \Omega$ and $\forall\left(q_{t}, q_{x}\right) \in D^{-} u(t, x)$, we have: $q_{t}+H\left(x, q_{x}\right) \leq 0$.

To prove this proposition, we will need the following lemma whose proof can be found in [15].

Lemma 3.6 (non-increasing functions). Let $v:[0, T] \mapsto \mathbb{R}$ be a lsc function such that

$$
D_{+} v(s)=\liminf _{h \rightarrow 0^{+}} \frac{v(s+h)-v(s)}{h} \leq 0 \quad \forall s \in[0, T) .
$$

Then $v$ is non-increasing on $[0, T]$.

Proof of Proposition 3.5. The proof will be split in five steps.

Step 1. Under assumptions (H2)-(H3), let us first prove the equivalence (i) $\Longleftrightarrow$ (ii).

Assume (i) and consider $t>0, \tau \in[0, t]$. Let $y_{x} \in S_{[0, \tau]}^{\Omega}(x)$, and define $v: \mathbb{R} \mapsto \mathbb{R}$ by

$$
v(s)=u\left(t-\tau+s, y_{x}(\tau-s)\right)
$$

We want to show that $v$ is non-increasing by using Lemma 3.6. For $s \in[0, \tau)$, we set $x_{1}=y_{x}(\tau-s)\left(\right.$ clearly, $x_{1}$ depends on $\tau$ and $s$ ). Thus we get:

$$
\begin{aligned}
D_{+} v(s) & =\liminf _{h \rightarrow 0^{+}} \frac{u\left(t-\tau+s+h, y_{x}(\tau-s-h)\right)-u\left(t-\tau+s, y_{x}(\tau-s)\right)}{h} \\
& =\liminf _{h \rightarrow 0^{+}} \frac{u\left(t-\tau+s+h, y_{x_{1}}(-h)\right)-u\left(t-\tau+s, x_{1}\right)}{h}=D_{\uparrow}^{y_{x_{1}}} u\left(t-\tau+s, x_{1}\right) \quad \forall s \in[0, \tau[
\end{aligned}
$$


where we have set $y_{x_{1}}(-h):=y_{x}(\tau-s-h)$ for $h$ small enough. This backward trajectory $y_{x_{1}}$ belongs to $S^{\Omega,-}\left(x_{1}\right)$, and thanks to (i), we deduce that $D_{+} v(s) \leq 0$ for $s \in[0, \tau)$. Therefore, Lemma 3.6 implies that $v$ is nonincreasing on $[0, \tau]$ and then $v(\tau) \leq v(0)$, which gives that

$$
u(t, x) \leq u\left(t-\tau, y_{x}(\tau)\right)
$$

Conversely, assume that (ii) holds true and let $(t, x) \in \mathbb{R}^{+} \times \mathbb{R}^{n}$. The case when $S^{\Omega,-}(x)=\emptyset$ is trivial. Let us consider that $S^{\Omega,-}(x) \neq \emptyset$ and let $y_{x} \in S^{\Omega,-}(x)$. There exists $\nu>0$ such that

$$
\dot{y}_{x}(s) \in F\left(y_{x}(s)\right) \text { on }[-\nu, 0] \text { and } \quad y_{x}(0)=x \text {. }
$$

For every $h \in[0, \nu]$, we define $x_{h}=y_{x}(-h)$ and consider the trajectory $y_{x_{h}}$ defined by: $y_{x_{h}}(s)=y_{x}(s-h)$ for $s \in[0, h]$. In particular, $y_{x_{h}}$ belongs to $S_{[0, h]}^{\Omega}\left(x_{h}\right)$. From (ii) we get:

$$
u\left(t+h, x_{h}\right) \leq u\left(t, y_{x_{h}}(h)\right)
$$

which gives

$$
u\left(t+h, y_{x}(-h)\right) \leq u(t, x)
$$

Therefore,

$$
D_{\uparrow}^{y_{x}} u(t, x)=\liminf _{h \rightarrow 0^{+}} \frac{u\left(t+h, y_{x}(-h)\right)-u(t, x)}{h} \leq 0,
$$

which proves assertion (i).

Step 2. From now on, we assume also that (H5) is fulfilled and that $\Omega$ is an open set. We first prove that (i) $\Longrightarrow$ (iii). Let $t \geq 0, x \in \Omega$ and $p \in F(x)$. Filippov's Theorem implies that there exists $y_{x} \in S^{\Omega,-}(x)$ (with $\left.y_{x}(0)=x\right)$ and such that

$$
\lim _{h \rightarrow 0^{+}} \frac{y_{x}(-h)-x}{h}=-p .
$$

Let $\left(h_{n}\right)_{n}$, with $h_{n} \rightarrow 0$, be a sequence of positive numbers such that:

$$
\lim _{n \rightarrow+\infty} \frac{u\left(t+h_{n}, y_{x}\left(-h_{n}\right)\right)-u(t, x)}{h_{n}}=D_{\uparrow}^{y_{x}} u(t, x) .
$$

For every $n \geq 0$, set $q_{n}:=-\frac{y_{x}\left(-h_{n}\right)-x}{h_{n}}$. Clearly, $q_{n}$ tends to $p$ when $n$ goes to $+\infty$. Moreover,

$$
D_{\uparrow} u(t, x)(1,-p)=\liminf _{\substack{h \rightarrow 0^{+} \\ q \rightarrow p}} \frac{u(t+h, x-h q)-u(t, x)}{h} \leq \lim _{n \rightarrow+\infty} \frac{u\left(t+h_{n}, x-h_{n} q_{n}\right)-u(t, x)}{h_{n}}=D_{\uparrow}^{y_{x}} u(t, x) .
$$

We deduce that for every $p \in F(x)$, we have $D_{\uparrow} u(t, x)(1,-p) \leq 0$ and (iii) holds true.

Step 3. The assertion (iii) $\Longrightarrow$ (ii) can be obtained by using similar arguments as in [16], Theorem 3.3. Let us point out that the paper [16] deals only with final state constraints $\left(\mathcal{K}=\mathbb{R}^{n}\right)$. However, the proof is still valid when we consider that the set of trajectories is in an open set $\Omega$. For the reader's convenience, we give the main steps of the proof. Let us assume (iii) and fix $t \geq 0, x \in \Omega, \tau \in[0, t]$, and $y_{x} \in S_{[0, \tau]}^{\Omega}(x)$. Now, we consider the application

$$
\mathcal{T}:[0, \tau] \ni s \longmapsto\left(\begin{array}{c}
t-\tau+s \\
y_{x}(\tau-s) \\
u\left(t-\tau, y_{x}(\tau)\right)
\end{array}\right)
$$


It is clear that $\mathcal{T}(0):=\left(t-\tau, y_{x}(\tau), u\left(t-\tau, y_{x}(\tau)\right)\right)$ belongs to the epigraph of $u$, denoted $\mathcal{E} p(u)$. On the other hand, by (iii) and thanks to [2], Proposition 6.1.4, we obtain that $(1,-p, 0)$ is a contingent ${ }^{5}$ direction to $\mathcal{E} p(u)$ at every $(\gamma, \xi, z) \in \mathbb{R}^{+} \times \Omega \times \mathbb{R}$ such that

$$
(\gamma, \xi, z) \in \mathcal{E} p(u) \text {, and } p \in F(\xi) \text {. }
$$

Therefore, $u$ being lsc, by using the viability theorem (see the proof of [16], Thm. 3.3), we get that $\mathcal{T}(s) \in \mathcal{E} p(u)$ for every $s \in[0, \tau]$. We deduce that:

$$
u\left(t-\tau+s, y_{x}(\tau-s)\right) \leq u\left(t-\tau, y_{x}(\tau)\right) \quad \forall s \in[0, \tau]
$$

which proves (ii).

Let us also remark that the same arguments give the following implication:

$$
\begin{aligned}
{\left[\forall t>0, \forall x \in \Omega, \max _{p \in F(x)} D_{\uparrow} u(t, x)(1,-p) \leq 0\right] } & \\
& \Longrightarrow\left[\forall t \geq 0, \forall x \in \Omega, \forall \tau \in\left[0, t\left[, \forall y_{x} \in S_{[0, \tau]}^{\Omega}(x), u(t, x) \leq u\left(t-\tau, y_{x}(\tau)\right)\right] .\right.\right.
\end{aligned}
$$

Indeed, due to the inversion of the time, the fact that we do not assume the left hand side property at initial time implies that we do not recover the right hand side at the final time $\tau=t$.

Step 4. Now we assume that (iii) is satisfied and we will prove that (iv) holds true. From the previous steps, we know that (i) and (ii) are also satisfied. Then assertion (iv)(b) can be deduced from (2.7) and (iii). It remains to prove assertion (iv)(a). For this, let us fix $x \in \Omega$. Let $\left(z_{i}\right)_{i} \subset \Omega$ and $\left.\left(t_{i}\right)_{i} \subset\right] 0,+\infty[$ such that:

$$
z_{i} \longrightarrow x, t_{i} \longrightarrow 0, \quad \text { and } u\left(t_{i}, z_{i}\right) \longrightarrow \liminf _{t \rightarrow 0^{+} z \rightarrow x} u(t, z) \quad \text { when } i \rightarrow+\infty .
$$

Let $\left(y_{i}\right)_{i}$ be a sequence of trajectories satisfying, for every $i \geq 1, \dot{y}_{i}(s)=F(y(s))$ on $\left(0, t_{i}\right)$ and $y_{i}\left(t_{i}\right)=x$. By Gronwall Lemma, we get $y_{i}(0) \rightarrow x$ when $i \rightarrow+\infty$. Moreover, by (ii) we have: $u\left(t_{i}, y_{i}(0)\right) \leq u(0, x)$. Therefore, since $u$ is lsc, we deduce:

$$
u(0, x) \leq \liminf _{t \rightarrow 0^{+} z \rightarrow x} u(t, z) \leq \lim _{i \rightarrow+\infty} u\left(t_{i}, y_{i}(0)\right) \leq u(0, x) .
$$

Step 5. Finally, we prove that (iv) $\Longrightarrow$ (ii). From [16], Lemma 4.4, we know that (iv)(b) is equivalent to the assertion

$$
\forall t>0, \forall x \in \Omega, \max _{p \in F(x)} D_{\uparrow} u(t, x)(1,-p) \leq 0,
$$

and by (3.20) we conclude that

$$
\forall t \geq 0, \forall x \in \Omega, \forall \tau \in\left[0, t\left[, \forall y_{x} \in S_{[0, \tau]}^{\Omega}(x), \quad u(t, x) \leq u\left(t-\tau, y_{x}(\tau)\right) .\right.\right.
$$

Now, it remains just to prove that the assertion (3.21) is still true up to time $\tau=t$. Let $t \geq 0, x \in \Omega$ and $y_{x} \in S_{[0, t]}^{\Omega}(x)$. Let $\left(z_{i}\right)_{i} \subset \Omega$ and $\left(t_{i}\right)_{i}$ a sequence of positive numbers such that:

$$
z_{i} \rightarrow y_{x}(t), \quad t_{i} \rightarrow 0^{+}, \quad u\left(t_{i}, z_{i}\right) \rightarrow u\left(0, y_{x}(t)\right) .
$$

Consider, for every $i \geq 1$, a trajectory $y^{i}$ satisfying $\dot{y}^{i}(s) \in F\left(y^{i}(s)\right), y^{i}\left(t-t_{i}\right)=z_{i}$ and $\left\|y^{i}-y_{x}\right\|_{L^{\infty}} \rightarrow 0$. The Gronwall Lemma yields that $y^{i}(0)$ converges to $x$. Moreover, since $\Omega$ is an open set, $y^{i} \subset \Omega$ on $\left[0, t_{i}\right]$ for $i$

\footnotetext{
${ }^{5}$ Let $K$ be a nonempty subset of $\mathbb{R}^{n}$ and $z \in K$. A vector $v$ in $\mathbb{R}^{n}$ is said a contingent direction to $K$ at $z$ if and only if: $\liminf _{h \rightarrow 0^{+}} d\left(v, \frac{K-z}{h}\right)=0$.
} 
big enough. Therefore, by (3.21), we obtain: $u\left(t, y^{i}(0)\right) \leq u\left(t_{i}, y^{i}\left(t-t_{i}\right)\right)=u\left(t_{i}, z^{i}\right)$. Since $u$ is lsc, by (iv)(a) we conclude:

$$
u(t, x) \leq \lim _{i \rightarrow \infty} u\left(t, y^{i}(0)\right) \leq \lim _{i \rightarrow \infty} u\left(t_{i}, z^{i}\right)=u\left(0, y_{x}(t)\right)
$$

which proves that (3.21) is true for $\tau=t$.

Corollary 3.7 (sub-optimality of the value function). Assume (H1)-(H4). Then, the value function $\vartheta$ satisfies for every $(t, x) \in \mathbb{R}^{+} \times \mathbb{R}^{n}$

$$
\sup _{y \in S^{\mathcal{K},-}(x)} D_{\uparrow}^{y} \vartheta(t, x) \leq 0 .
$$

Proof. This is a consequence of Propositions 3.1(ii) and 3.5.

\subsection{Proof of Theorem 2.4}

The equivalence between (ii) and (iii) follows from Propositions 3.2 and 3.5. Remark that Proposition 3.2 is state for $x \in \mathbb{R}^{n}$ while Theorem 2.4 (ii) and (iii) is state for $x \in \mathcal{K}$. Nevertheless, these equations are trivially satisfied for $x \in \mathbb{R}^{n} \backslash \mathcal{K}$ (since $u=1$ in $\mathbb{R}^{n} \backslash \mathcal{K}$ ).

We now prove the equivalence between (i) and (ii). The fact that the value function $\vartheta$ satisfies (2.8) follows from Corollary 3.4 and 3.7 and from the definition of $\vartheta$.

Conversely, let us assume that $u$ satisfies (2.8) and let us prove that $u=\vartheta$. Let $x \in \mathcal{K}$ and $t>0$. The proof is splitted in two steps:

Step 1: $u(t, x) \geq \vartheta(t, x)$.

Indeed, using Proposition 3.2, we get that there exists $y_{x} \in S_{[0, t]}(x)$ such that:

$$
u\left(t-\tau, y_{x}(\tau)\right) \leq u(t, x) \quad \forall \tau \in[0, t] .
$$

Two cases may occur. In the first case, $y_{x}$ does not belong to $S_{[0, t]}^{\mathcal{K}}(x)$ which means that there exists $\tau \in[0, t]$ with $y_{x}(\tau) \notin \mathcal{K}$. In this case, we have

$$
u\left(t-\tau, y_{x}(\tau)\right)=1 \leq u(t, x) .
$$

Since $u, \vartheta$ are bounded from above by 1 , we conclude that $u(t, x)=1$ and then

$$
u(t, x) \geq \vartheta(t, x) .
$$

On the other hand, if $y_{x}$ belongs to $S_{[0, t]}^{\mathcal{K}}(x)$ then by taking the limit $\tau \rightarrow t^{-}$in (3.22) and using the fact that $u$ is lsc, we get

$$
\vartheta(t, x) \leq \varphi\left(y_{x}(t)\right) \leq \liminf _{\tau \rightarrow t^{-}} u\left(t-\tau, y_{x}(\tau)\right) \leq u(t, x) .
$$

Step 2: $\vartheta(t, x) \geq u(t, x)$.

It suffices to consider the case $S_{[0, t]}^{\mathcal{K}}(x) \neq \emptyset$ (otherwise $\vartheta(t, x)=1 \geq u(t, x)$ ). In this case, by Proposition 3.1, there exists an admissible trajectory $y_{x} \in S_{[0, t]}^{\mathcal{K}}(x)$ such that

$$
\varphi\left(y_{x}(t)\right)=\vartheta\left(0, y_{x}(t)\right)=\vartheta(t, x) .
$$

On the other hand, by Proposition 3.5, we also have that

$$
u(t, x) \leq u\left(0, y_{x}(t)\right)=\varphi\left(y_{x}(t)\right) .
$$

This implies that $\vartheta(t, x) \geq u(t, x)$ and ends the proof of the theorem. 


\section{Some Particular Situations}

The goal of this section is to show that Theorem 2.4 leads to a characterisation of the value function in term of Hamilton-Jacobi equations when a qualification condition is satisfied and $F$ is continuous. In particular, we can recover the results of Soner [21] (see also [19]) when inward qualification condition holds (see Sect. 4.1) and also the results of Frankowska et al. $[17,18]$ in the case when outward qualification condition is satisfied (see Sect. 4.2).

In this section, we limit our attention to state constraint sets $\mathcal{K}$ expressible as

$$
\mathcal{K}=\bigcap_{j=1}^{r}\left\{x, h_{j}(x) \leq 0\right\}, \quad \stackrel{\circ}{\mathcal{K}}=\bigcap_{j=1}^{r}\left\{x, h_{j}(x)<0\right\}
$$

for a finite family of $C^{1,1}$ functions ${ }^{6}\left\{h_{j}: \mathbb{R}^{n} \rightarrow \mathbb{R}\right\}_{j=1, \ldots, r}$. We also denote by

$$
I(x)=\left\{j \in\{1, \ldots, r\}, h_{j}(x)=0\right\}
$$

the active set of index values at a point $x \in \partial \mathcal{K}$. We assume throughout this section that $\stackrel{\circ}{\mathcal{K}} \neq \emptyset$.

\subsection{Inward pointing condition}

In [21], Soner introduced the "inward-pointing" constraint qualification:

$$
\exists \beta>0, \forall x \in \partial \mathcal{K}, \quad \min _{p \in F(x)} p \cdot \nabla_{x} h_{j}(x)<-\beta \quad \forall j \in I(x) .
$$

Under this "controllability" hypothesis, Soner proved that the value function is continuous and is the unique solution of a constrained HJB equation in an appropriate sense. In smooth cases (with $\varphi$ Lipschitz continuous), this viscosity notion can be interpreted as solution of a Neumann boundary equation (see the paper of CapuzzoDolcetta and Lions [13]). Generalizations of this condition to the case of a state constraints set $\mathcal{K}$ with nonsmooth boundary, were introduced by Ishii and Koike [19]. They also modified the notion of solutions, by modifying the Hamiltonian.

Condition (4.24) is a strengthened viability condition [1]. Geometrically, (4.24) means that from each point $x \in \partial \mathcal{K}$ a trajectory enters into $\stackrel{\circ}{\mathcal{K}}$, while the viability condition guarantees that a solution stays in $\mathcal{K}$ forever. Moreover, under condition (4.24), each trajectory starting at $x$ on the boundary can be approximated by a sequence of trajectories lying in the interior of $\mathcal{K}$. More precisely, we have the following lemma.

Lemma 4.1. Assume that $F$ satisfies $(\mathrm{H} 2)-(\mathrm{H} 5)$ and $\mathcal{K}$ is defined as in $(4.23)$. Let $y_{x} \in S_{[0, T]}^{\mathcal{K}}(x)$ for $(T, x) \in$ $\mathbb{R}^{+} \times \mathcal{K}$. Let $\left(t_{i}, x_{i}\right) \in \mathbb{R}_{*}^{+} \times \stackrel{\circ}{\mathcal{K}}$ be such that $\left(t_{i}, x_{i}\right) \rightarrow(0, x)$. If $(4.24)$ holds true, then there exists a sequence of trajectories $y^{i}$ such that

$$
\dot{y}^{i}(t) \in F\left(y^{i}(t)\right) \text { for } t \in\left[t_{i}, T\right], \quad y^{i}\left(t_{i}\right)=x_{i}, \quad y^{i}(t) \in \stackrel{\circ}{\mathcal{K}} \text { on }\left[t_{i}, T\right]
$$

and

$$
\left\|y^{i}-y_{x}\right\|_{L^{\infty}\left(\left[t_{i}, T\right] ; \mathbb{R}^{n}\right)} \rightarrow 0
$$

The proof of this lemma can be obtained by similar arguments as in [18], Lemma 4.1.

Theorem 4.2 (characterization of the value function under inward-pointing constraints). Let $\mathcal{K}$ be given by (4.23). Assume that $\varphi$ is a continuous function on $\mathcal{K}$. Assume also that (H1)-(H5) and (4.24) are satisfied.

\footnotetext{
${ }^{6} C^{1,1}$ being the class of $C^{1}$-functions with Lipschitz continuous gradients.
} 
Let $u: \mathbb{R}^{n} \times \mathbb{R} \mapsto\left[-M_{0}, M_{0}\right] \cup\{1\}$ be a continuous function such that $u=1$ on $\mathcal{K}^{c}$. Then, the following assertions are equivalent:

(i) $u=\vartheta$.

(ii) $\left\{\begin{array}{l}u(0, x)=\varphi(x), \\ \forall t>0, x \in \mathcal{K}, \sup _{p \in F(x)}-D_{\uparrow} u(t, x)(-1, p) \geq 0, \\ \forall t \geq 0, x \in \stackrel{\circ}{\mathcal{K}}, \sup _{p \in F(x)} D_{\uparrow} u(t, x)(1,-p) \leq 0 .\end{array}\right.$

(iii) $\begin{cases}u(0, x)=\varphi(x), & \\ \partial_{t} u(t, x)+H\left(x, D_{x} u(t, x)\right) \geq 0 & \text { for } t>0, x \in \mathcal{K}, \\ \partial_{t} u(t, x)+H\left(x, D_{x} u(t, x)\right)=0 & \text { for } t>0, x \in \mathcal{K} .\end{cases}$

Proof. This theorem is already proved in [22]. It can be also deduced from Theorem 2.4. Indeed, under inward qualification constraint (4.24), when $\varphi$ is continuous on $\mathcal{K}$, the value function is also continuous on $\mathcal{K}$. Moreover, for every continuous function $u: \mathbb{R}^{+} \times \mathbb{R}^{n} \rightarrow \mathbb{R}$, we have:

$$
\left[\forall t \geq 0, \forall x \in \mathcal{K}, \sup _{y \in S^{\circ},-(x)} D_{\uparrow}^{y} u(t, x) \leq 0\right] \Longrightarrow\left[\forall t \geq 0, \forall x \in \mathcal{K}, \sup _{y \in S^{\mathcal{K},-},(x)} D_{\uparrow}^{y} u(t, x) \leq 0\right],
$$

which means that the information about the behavior of $u$ along trajectories arriving at the boundary of $\mathcal{K}$ can be deduced from the behavior along trajectories lying in the interior of $\mathcal{K}$. The proof of this claim is mainly based on Lemma 4.1.

Assume that the left hand side of $(4.25)$ is satisfied. Let $(t, x)$ be in $\mathbb{R}^{+} \times \partial \mathcal{K}$. Let $y_{x}$ be a trajectory in $S^{\mathcal{K},-}(x)$. Then there exists $\nu>0$ such that $y_{x}([-\nu, 0]) \subset \mathcal{K}$ and $y_{x}(0)=x$.

If $y_{x}([-\nu, 0[) \subset \mathcal{K}$, then by using Proposition 3.5, we deduce for every $h \in] 0, \nu]$ and every $\left.\left.\tau \in\right] 0, h\right]$ :

$$
u\left(t+h, y_{x}(-h)\right) \leq u\left(t+\tau, y_{x}(-\tau)\right) .
$$

By sending $\tau$ to 0 , and using the continuity of $u$, we get:

$$
\left.\left.u\left(t+h, y_{x}(-h)\right) \leq u(t, x) \quad \forall h \in\right] 0, \nu\right],
$$

which implies that $D_{\uparrow}^{y_{x}} u(t, x) \leq 0$.

In the general situation, for $h \in[0, \nu]$, we use Lemma 4.1 to approach $y_{x}$ on $[-h, 0]$ by a sequence of

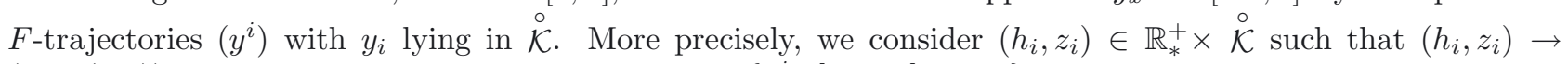
$\left(h, y_{x}(-h)\right)$. By Lemma 4.1, there exists a sequence $\left\{y^{i}:\left[-h_{i}, 0\right] \rightarrow \mathbb{R}^{n}\right\}$ such that

$$
y^{i}\left(-h_{i}\right)=z_{i}, \quad y^{i} \subset \mathcal{K} \quad \text { on }\left[-h_{i}, 0\right] \quad \text { and } \quad\left\|y^{i}-y_{x}\right\|_{L^{\infty}\left(\left[-h_{i}, 0\right] ; \mathbb{R}^{n}\right)} \rightarrow 0 .
$$

Using (4.26) (applied to $y^{i} \in S^{\stackrel{\mathcal{K}}{,},-}\left(y^{i}(0)\right)$ ), we deduce that

$$
u\left(t+h_{i}, z_{i}\right) \leq u\left(t, y^{i}(0)\right) .
$$

Sending, $i \rightarrow \infty$, we deduce that

$$
u\left(t+h, y_{x}(-h)\right) \leq u(t, x),
$$

for every $h \in[0, \nu]$, which implies that $D_{\uparrow}^{y_{x}} u(t, x) \leq 0$. 
Remark 4.3. It is clear that assertion (4.25) amounts to saying that, under the inward condition (4.24), if $u$ is suboptimal along any trajectory $y_{x}$ lying in the interior $\stackrel{\circ}{\mathcal{K}}$ then it is also suboptimal along any trajectory remaining in the closed set $\mathcal{K}$.

\subsection{Outward pointing condition}

In $[17,18]$, Frankowska et al. introduced another condition to study the characterization of the value function:

$$
\forall x \in \partial \mathcal{K}, \quad \max _{p \in F(x)} p \cdot \nabla h_{j}(x)>0 \quad \forall j \in I(x) .
$$

This condition said "outward pointing qualification" can be seen as a reverse to Soner's assumption (4.24). It means that from each boundary point $x \in \mathcal{K}$, a trajectory solution of (2.3) leaves immediately $\mathcal{K}$. It means also that each point of the boundary can be hit by a trajectory coming from the interior of $\mathcal{K}$. Under assumption (4.27), a similar property to Lemma 4.1 is the following (see [18], Lem. 4.1):

Lemma 4.4. Assume (H2)-(H5) and (4.27) hold true. Let $T>0, x \in \mathcal{K}$ and $y_{x} \in S^{\mathcal{K},-}(x)$ such that:

$$
\dot{y}_{x}(t) \in F\left(y_{x}(t)\right) \quad \text { for } t \in[-T, 0] .
$$

Let $\left(t_{i}, x_{i}\right) \in \mathbb{R}_{*}^{+} \times \stackrel{\circ}{\mathcal{K}}$ be a sequence satisfying $\left(t_{i}, x_{i}\right) \rightarrow(0, x)$, when $i \rightarrow+\infty$. Then there exists a sequence of trajectories $y^{i}$ such that

$$
\dot{y}^{i}(t) \in F\left(y^{i}(t)\right) \text { for } t \in\left[-T,-t_{i}\right], \quad y^{i}\left(-t_{i}\right)=x_{i}, \quad y^{i}(t) \in \stackrel{\circ}{\mathcal{K}} \text { on }\left[-T,-t_{i}\right]
$$

and

$$
\left\|y^{i}-y_{x}\right\|_{L^{\infty}\left(\left[-T,-t_{i}\right] ; \mathbb{R}^{n}\right)} \rightarrow 0 .
$$

Here, under the outward condition (4.27), any trajectory arriving to a boundary point $x \in \partial K$ can be approximated by a sequence of trajectories lying inside $\mathcal{K}$, while this approximation is claimed, under the inward condition, for a trajectory starting at the boundary.

Under assumption (4.27), the value function is not necessarily continuous, but is still the unique solution of HJB equation in a lsc sense in lines of $[10,16]$.

Theorem 4.5 (characterization of the value function under outward-pointing constraints). Let $u: \mathbb{R}^{n} \times \mathbb{R} \mapsto$ $\left[-M_{0}, M_{0}\right] \cup\{1\}$ be a lsc function such that $u=1$ on $\mathcal{K}^{c}$. We assume that $(\mathrm{H} 1)-(\mathrm{H} 5)$ and $(4.27)$ hold. Then the following assertions are equivalent:

(i) $u=\vartheta$.

(ii) $\left\{\begin{array}{l}\forall x \in \mathcal{K}, \liminf _{\substack{s \rightarrow 0^{+}, z \rightarrow x}} u(s, z)=u(0, x)=\varphi(x), \\ \forall t>0, \forall x \in \mathcal{K}, \liminf _{\substack{s \rightarrow t^{+}, \mathcal{K} \ni z \rightarrow x}} u(s, z)=u(t, x), \\ \forall t>0, x \in \mathcal{K}, \sup _{p \in F(x)}-D_{\uparrow} u(t, x)(-1, p) \geq 0, \\ \forall t \geq 0, x \in \mathcal{K}, \sup _{p \in F(x)} D_{\uparrow} u(t, x)(1,-p) \leq 0 .\end{array}\right.$ 


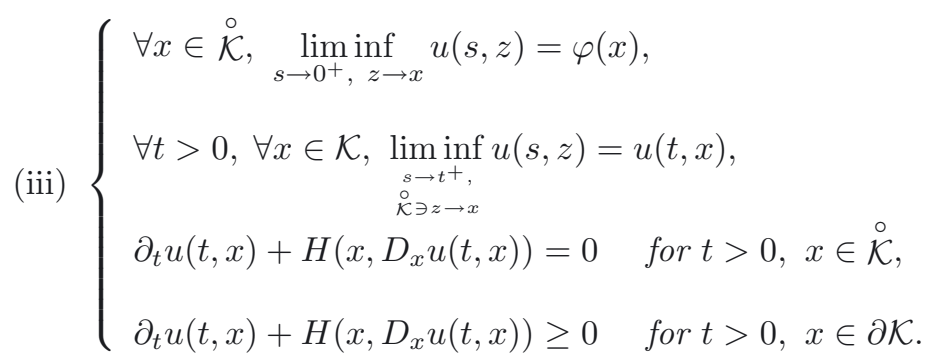

Proof. The equivalence between (ii) and (iii) follows from Propositions 3.2 and 3.5. The fact that (i) implies (ii) follows from Theorem 2.4 and the fact that, under the outward-pointing constraint, the value function $\vartheta$ satisfies the second line of (ii) [18].

For the implication (ii) $\Rightarrow$ (i), using Theorem 2.4 and Proposition 3.5, we just have to prove that

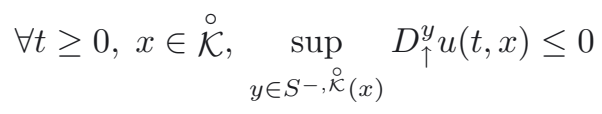

implies that

$$
\forall t \geq 0, x \in \mathcal{K}, \sup _{y \in S^{-, \mathcal{K}}(x)} D_{\uparrow}^{y} u(t, x) \leq 0 .
$$

Let $t \geq 0, x \in \mathcal{K}$ and $y \in S^{\mathcal{K},-}(x) \backslash S^{\stackrel{\circ}{\mathcal{K}},-}(x)$.

Consider $\left(t_{i}, x_{i}\right) \in \mathbb{R}_{*}^{+} \times \stackrel{\circ}{\mathcal{K}}$ such that:

$$
\left(t_{i}, x_{i}\right) \rightarrow(0, x) \text { and } u\left(t_{i}, x_{i}\right) \rightarrow u(t, x) \quad \text { when } i \rightarrow+\infty \text {. }
$$

By Lemma 4.4, there exists a sequence $\left\{y^{i}:\left[-\tau,-t_{i}\right] \rightarrow \mathbb{R}^{n}\right\}$ such that

$$
y^{i}\left(-t_{i}\right)=x_{i}, \quad y^{i} \in \stackrel{\circ}{\mathcal{K}} \text { on }\left[-\tau,-t_{i}\right] \quad \text { and } \quad\left\|y^{i}-y_{x}\right\|_{L^{\infty}\left(\left[-\tau,-t_{i}\right] ; \mathbb{R}^{n}\right)} \rightarrow 0
$$

We define $\tilde{y}^{i}(\tau)=y^{i}\left(\tau+t_{i}\right) \in S_{\left[-\tau+t_{i}, 0\right]}^{\stackrel{\circ}{\mathcal{K},-}}\left(x_{i}\right)$. By (4.28), we deduce that

$$
\liminf _{h \rightarrow 0} \frac{u\left(t+h, \tilde{y}^{i}(-h)\right)-u\left(t, x_{i}\right)}{h} \leq 0
$$

Sending $i \rightarrow \infty$, using the fact that $u$ is lsc and that $u\left(t, x_{i}\right) \rightarrow u(t, x)$, we get that

$$
\liminf _{h \rightarrow 0} \frac{u\left(t+h, \tilde{y}_{x}(-h)\right)-u(t, x)}{h} \leq 0
$$

which gives (4.29).

\section{Relaxation of State COnstraints}

Consider $\left(\mathcal{K}_{\varepsilon}\right)_{\varepsilon>0}$ a sequence of subsets of $\mathbb{R}^{n}$ such that

(i) for every $\varepsilon>0, \mathcal{K}_{\varepsilon}$ is closed and $\mathcal{K} \subset \mathcal{K}_{\varepsilon}$,

(ii) $\mathcal{K}_{\eta} \subset \stackrel{\circ}{\mathcal{K}}_{\varepsilon}$ for every $\varepsilon>0$ and $\eta \in(0, \varepsilon)$,

(iii) $\forall x \in \mathcal{K}, \lim _{\varepsilon \rightarrow 0} d\left(x, \mathbb{R}^{n} \backslash \mathcal{K}_{\varepsilon}\right)=0$, 
where $d$ is a distance function. For instance, we may simply consider $\mathcal{K}_{\varepsilon}:=\left\{x \in \mathbb{R}^{n}, d(x, K) \leq \varepsilon\right\}$. In all the sequel, for every $t \geq 0$, we shall say that $y_{x}$ is a $\mathcal{K}_{\varepsilon}$-admissible trajectory on $[0, t]$ when $y_{x}$ belongs to $S_{[0, t]}^{\mathcal{K}_{\varepsilon}}(x)$.

Our aim in this section is to prove Theorem 2.8, that is, to prove the pointwise convergence of $u_{\varepsilon}(t, x)$ towards $\vartheta(t, x), u_{\varepsilon}: \mathbb{R}^{+} \times \mathbb{R}^{n} \mapsto\left[-M_{0}, M_{0}\right] \cup\{1\}$ being a solution of (2.10).

Notice that (2.10) admits (at least) one solution $u_{\varepsilon}$. Indeed, the value function associated to a control problem (2.5) with state constraints in $\mathcal{K}_{\varepsilon}$ (instead of $\mathcal{K}$ ) is a solution of (2.10). However, this equation may admit several solutions. The main feature of Theorem 2.8 is that, by enlarging the set $\mathcal{K}$ and considering (2.10), we neither have to deal with difficult boundary constraints, nor we have to require any constraint qualification assumption on $\partial \mathcal{K}_{\varepsilon}$. We just take any solution $u_{\varepsilon}$ of $(2.10)$ on $\mathcal{K}_{\varepsilon}$ and then by sending $\varepsilon$ to 0 , this solution $u_{\varepsilon}$ will converge to the value function $\vartheta$ (which is the unique solution of the HJB equation (2.9a)-(2.9b) and satisfying the boundary conditions $(2.9 \mathrm{c})-(2.9 \mathrm{e}))$.

Proof of Theorem 2.8. To prove the result we proceed in several steps.

Step 1. For $t>0$ and $x \in \mathcal{K}$ given, we claim that $\liminf _{\varepsilon \rightarrow 0} u_{\varepsilon}(t, x) \geq \vartheta(t, x)$.

Let us set $\ell:=\liminf _{\varepsilon \rightarrow 0} u_{\varepsilon}(t, x)$, and assume that $\ell<1$ (otherwise the result is immediate). We consider a subsequence $\varepsilon_{k}>0$ such that $\varepsilon_{k} \rightarrow 0$ (with $\varepsilon_{k} \leq 1$ ) and (for $k$ large enough)

$$
1>\ell+\frac{1}{k} \geq u_{\varepsilon_{k}}(t, x) .
$$

For $x \in \mathbb{R}^{n} \backslash \mathcal{K}_{\varepsilon_{k}}$ and $t>0$, we have $u_{\varepsilon_{k}} \equiv 1$ locally around $(t, x)$ and thus (2.10a) is also satisfied at the point $(t, x)$ for any $t>0$ and $x \in \mathbb{R}^{n}$.

Therefore we can apply Proposition 3.2 (using that (iii) $\Rightarrow$ (ii)) and obtain that there exists $y_{x}^{\varepsilon_{k}} \in S_{[0, t]}(x)$ such that $\forall \tau \in[0, t], u_{\varepsilon_{k}}\left(t-\tau, y_{x}^{\varepsilon_{k}}(\tau)\right) \leq u_{\varepsilon_{k}}(t, x)$. We then deduce that

$$
\ell+\frac{1}{k} \geq u_{\varepsilon_{k}}(t, x) \geq \varphi\left(y_{x}^{\varepsilon_{k}}(t)\right) .
$$

By Remark 2.2, we can extract from $y_{x}^{\varepsilon_{k}}$ a convergent subsequence towards some trajectory $y_{x} \in S_{[0, t]}(x)$. At the limit, when $k$ tends to $+\infty$, and taking into account that $\varphi$ is lsc, we obtain

$$
\ell \geq \varphi\left(y_{x}(t)\right)
$$

On the other hand, using that $u_{\varepsilon_{k}} \equiv 1$ on $\mathbb{R}^{n} \backslash \mathcal{K}_{\varepsilon_{k}}$ and the fact that $u_{\varepsilon_{k}}(t, x)<1$, we deduce that $y_{x}^{\varepsilon_{k}}$ is $\mathcal{K}_{\varepsilon_{k}}$-admissible for every $k>0$. We then obtain that $y_{x} \in S_{[0, t]}^{\mathcal{K}}(x)$ by using assumption (H6)(iii) and the fact that $y_{x} \in S_{[0, t]}^{\mathcal{K}_{\varepsilon_{k}}}(x), \forall k>0$. This proves that

$$
\vartheta(t, x) \leq \varphi\left(y_{x}(t)\right) \leq \ell
$$

and concludes the proof of Step 1.

Step 2. We now claim that $\vartheta(t, x) \geq u_{\varepsilon}(t, x)$ for all $t>0, x \in \mathcal{K}$ and $\varepsilon>0$. To show this, let us consider $V_{\varepsilon}$, the value function of the optimal control problem with the relaxed constraint set $\mathcal{K}^{\varepsilon}$ instead of $\mathcal{K}$ :

$$
V_{\varepsilon}(t, x):= \begin{cases}\inf \left\{\varphi\left(y_{x}(t)\right), y_{x} \in S_{[0, t]}^{\mathcal{K}_{\varepsilon}}(x)\right\}, & \text { if } S_{[0, t]}^{\mathcal{K}_{\varepsilon}}(x) \neq \emptyset, \\ 1 & \text { otherwise. }\end{cases}
$$

By definition, for all $\varepsilon>0$, we have

$$
V_{\varepsilon} \leq \vartheta
$$

We have also the following lemma which proof is postponed. 
Lemma 5.1. Let $u_{\varepsilon}$ satisfy (2.10b), (2.10c) and (2.10d). For any $\eta \in(0, \varepsilon)$, we have

$$
u_{\varepsilon}(t, x) \leq V_{\eta}(t, x) .
$$

Combining Lemma 5.1 and (5.32) we obtain that $\vartheta(t, x) \geq V_{\frac{\varepsilon}{2}}(t, x) \geq u_{\varepsilon}(t, x)$.

Step 3. Conclusion: Combining Step 1 and Step 2, we get that

$$
\vartheta(t, x) \geq \limsup _{\varepsilon \rightarrow 0} u_{\varepsilon}(t, x) \geq \liminf _{\varepsilon \rightarrow 0} u_{\varepsilon}(t, x) \geq \vartheta(t, x) .
$$

This ends the proof of Theorem 2.8 .

Proof of Lemma 5.1. Let $\eta$ be in $(0, \varepsilon)$. Using that $u_{\varepsilon}$ satisfies Proposition 3.5(iv) with $\Omega:=\dot{\mathcal{K}}_{\varepsilon}$, we deduce by the same proposition that $u_{\varepsilon}$ is increasing along $\mathcal{K}_{\varepsilon}$-admissible trajectories, and in particular,

$$
\forall t \geq 0, \forall x \in \stackrel{\circ}{\mathcal{K}}_{\varepsilon}, \forall y_{x} \in S_{[0, t]}^{\stackrel{\circ}{\varepsilon}_{\varepsilon}}, u(t, x) \leq \varphi\left(y_{x}(t)\right) .
$$

Now, for any $\mathcal{K}_{\eta}$-admissible trajectory denoted $y_{x}^{\eta}(t)$, and since $\mathcal{K}_{\eta} \subset \mathcal{K}_{\varepsilon}$ (by (H6)(iii)), we can apply (5.33) and obtain that $u_{\varepsilon}(t, x) \leq \varphi\left(y_{x}^{\eta}(t)\right)$. Hence $u_{\varepsilon}(t, x) \leq V_{\eta}(t, x)$.

As claimed in Proposition 2.9, Equation (2.10) is equivalent to an HJB equation with Dirichlet boundary conditions. Let us prove this assertion.

Proof of Proposition 2.9. The equivalence of (i) and (ii), for the initial condition aspect as well as for the bilateral solution aspect in the interior of $\mathcal{K}_{\varepsilon}$ is easily obtained from Definitions 2.6 and 2.10.

We also obtain directly from (2.10a) that Definition 2.10(ii) holds, which concludes the proof of (i) $\Rightarrow$ (ii).

Conversely, there remains to show that if we assume (ii) then (2.10a) holds for $t>0$ and $x \in \partial \mathcal{K}_{\varepsilon}$. In the case when $u(t, x)=1$, since $u$ is lsc and takes its values in $\left[-M_{0}, M_{0}\right] \cup\{1\}$, we know that $u \equiv 1$ locally around $(t, x)$ and thus (2.10a) is also satisfied at $(t, x)$. Otherwise, $u(t, x)<1$, and thus Definition 2.10(ii) implies that

$$
p_{t}+\max _{p \in F(x)}\left(-p \cdot p_{x}\right) \geq 0
$$

for any $\left(p_{t}, p_{x}\right) \in D^{-} u(t, x)$, which gives the desired result.

We now give a result that can be useful for the approximation of backward reachable sets (see below). Let $d_{H}(A, B)$ be the Hausdorff distance between two compact sets $A, B$ of $\mathbb{R}^{n}$. Let $\lambda<1$ and

$$
\mathcal{D}(\lambda):=\{x, \varphi(x) \leq \lambda\} .
$$

For $t \geq 0$ given, we define the set $A_{t}(\lambda)$ by:

$$
A_{t}(\lambda):=\left\{x \in \mathbb{R}^{n}, \vartheta(t, x) \leq \lambda\right\}
$$

and, for a given $u_{\varepsilon}$ solution of $(2.10)$, the set $A_{t}^{\varepsilon}(\lambda)$ by:

$$
A_{t}^{\varepsilon}(\lambda):=\left\{x \in \mathbb{R}^{n}, u_{\varepsilon}(t, x) \leq \lambda\right\} .
$$

We have $A_{t}(\lambda) \subset A_{t}^{\varepsilon}(\lambda)$ since $u_{\varepsilon} \leq \vartheta$. Hence $A_{t}(\lambda) \subset \cap_{\varepsilon>0} A_{t}^{\varepsilon}(\lambda)$. The following shows that the reverse inclusion is true.

\footnotetext{
${ }^{7} d_{H}(A, B):=\max (\delta(A, B), \delta(B, A))$ where $\delta(A, B):=\max _{x \in A} d(x, B)$.
} 
Proposition 5.2. We assume (H1)-(H6). We have:

(i) $\cap_{\varepsilon>0} A_{t}^{\varepsilon}(\lambda)=A_{t}(\lambda)$.

(ii) If $\mathcal{D}(\lambda)$ is a compact set, then $\lim _{\varepsilon \rightarrow 0} d_{H}\left(A_{t}^{\varepsilon}(\lambda), A_{t}(\lambda)\right)=0$.

Proof. (i) is a simple consequence of Theorem 2.8 .

We now prove (ii). For a given $\lambda$, let us simply denote $A_{t}$ and $A_{t}^{\varepsilon}$ for $A_{t}(\lambda)$ and $A_{t}^{\varepsilon}(\lambda)$. We first notice that $A_{t} \subset A_{t}^{\varepsilon}$. Hence there remains to control

$$
\delta\left(A_{t}^{\varepsilon}, A_{t}\right):=\max _{x \in A_{t}^{\varepsilon}} d\left(x, A^{t}\right) .
$$

$A_{t}^{\varepsilon}$ is a closed set, and there exists $x_{\varepsilon} \in A_{t}^{\varepsilon}$ such that $\max _{x \in A_{t}^{\varepsilon}}\left(x, A_{t}\right):=d\left(x_{\varepsilon}, A_{t}\right)$. We consider a subsequence $\varepsilon_{n} \rightarrow 0$ such that $\left(d\left(x_{\varepsilon_{n}}, A_{t}\right)\right)$. Since $\mathcal{D}(\lambda)$ and $F$ are bounded, $A_{t}^{\varepsilon}$ is also a bounded set which contains $x_{\varepsilon_{n}}$ (for $\varepsilon_{n} \leq 1$ ). Then, we can extract from $x_{\varepsilon_{n}}$ a converging subsequence (still denoted $x_{\varepsilon_{n}}$ ) to some limit $x$.

Since $x_{\varepsilon_{n}} \in A_{t}^{\varepsilon}$ for all $\varepsilon_{n} \leq \varepsilon$, passing to the limit we obtain $x \in A_{t}^{\varepsilon}$ for any given $\varepsilon>0$. By Theorem 2.8 , we deduce that $x \in A_{t}$, and that $\left(d\left(x_{\varepsilon_{n}}, A_{t}\right)\right) \rightarrow 0$. This finally proves that all the sequence $\left(d\left(x_{\varepsilon}, A_{t}\right)\right)$ converges to 0 as $\varepsilon \rightarrow 0$.

Remark 5.3. A direct application is the following. Let $\mathcal{C}$ be a given target, that is a non empty compact set. We choose $\varphi(x):=d_{\mathcal{C}}(x)$, that is, the signed distance function to the set $\mathcal{C}$, and set $\lambda:=0$. Then it is easy to see that $A_{t}(0)$ corresponds to the backward reachable set at time $t$, that is,

$$
A_{t}(0)=\left\{x \in \mathbb{R}^{n}, \exists y_{x} \in S_{[0, t]}^{K}(x), \text { such that } y_{x}(t) \in \mathcal{C}\right\}
$$

Hence Proposition 5.2 gives an approximation result for the backward reachable set $A_{t}(0)$ by the sets $A_{t}^{\varepsilon}(0)$ associated to any $u_{\varepsilon}$ solution of $(2.10)$.

\section{A penalization APPROACH FOR STATE CONSTRAints PROBlems}

In the previous sections, we were interested by various characterization of the discontinuous value function $\vartheta(t, x)$. Now, we are interested by an approximation of $\vartheta$ by continuous functions. This approach is also closely related to our recent work [12] used for the characterization of reachable sets.

As before, the problem is to deal with general state constraints without using any controllability assumption. We recall that by $(\mathrm{H} 1) \varphi$ is a lsc function and $-M_{0} \leq \varphi(x) \leq M_{0}$ on $\mathcal{K}$.

Let us consider a sequence of Lipschitz continuous functions $g_{\varepsilon}: \mathbb{R}^{n} \rightarrow \mathbb{R}$ satisfying

$$
\begin{aligned}
& \text { (i) } g_{\varepsilon}(x):=-M_{0} \quad \forall x \in \mathcal{K}, \\
& \text { (ii) } g_{\varepsilon}(x) \leq 1 \quad \forall x \in \mathbb{R}^{n} \text {, and } \forall \delta>0, \quad \lim _{\varepsilon \rightarrow 0} \inf _{\{z: d(z, \mathcal{K}) \geq \delta\}} g_{\varepsilon}(z)=1 .
\end{aligned}
$$

For instance, $g_{\varepsilon}$ can be defined as in $(2.14)$. We consider $\vartheta_{\varepsilon}$ defined as follows:

$$
\vartheta_{\varepsilon}(t, x):=\inf _{y_{x} \in S_{[0, t]}(x)} \max \left(\varphi\left(y_{x}(t)\right), \max _{\theta \in[0, t]} g_{\varepsilon}\left(y_{x}(\theta)\right)\right) .
$$

Here the state variable is free, but the penalized cost function takes value 1 whenever the trajectory exits the set $\mathcal{K}$. Furthermore, by using similar arguments as in Barron and Jensen [10] and Barron [9] (see also Bokanowski et al. [12]), we can easily prove that $\vartheta_{\varepsilon}$ is the unique solution of an HJB equation:

Proposition 6.1. Under assumptions $(\mathrm{H} 1)-(\mathrm{H} 5)$, for every $\varepsilon>0$, the function $\vartheta_{\varepsilon}$ is the unique lsc viscosity solution of the following inequation:

$$
\begin{aligned}
& \min \left(\partial_{t} \vartheta_{\varepsilon}+H\left(x, \nabla \vartheta_{\varepsilon}\right), \vartheta_{\varepsilon}-g_{\varepsilon}(x)\right)=0, \quad t>0, x \in \mathbb{R}^{n}, \\
& \vartheta_{\varepsilon}(0, x)=\max \left(\varphi(x), g_{\varepsilon}(x)\right), \quad x \in \mathbb{R}^{n} .
\end{aligned}
$$


Now, the proof of Theorem 2.12 becomes very clear. It suffices to prove that the value function $\vartheta_{\varepsilon}$ converges pointwisely to $\vartheta$. This characterization is very useful in order to compute numerically an approximation of $\vartheta$.

Proof of Theorem 2.12. First let us remark that

$$
\vartheta(t, x)=\inf _{y_{x} \in S_{[0, t]}(x)} \max \left(\varphi\left(y_{x}(t)\right), \max _{\theta \in[0, t]} g_{0}\left(y_{x}(\theta)\right)\right)
$$

where $g_{0}(x)=M_{0}$ for $x \in \mathcal{K}$ and 1 elsewhere. Thus for every $(t, x) \in \mathbb{R}^{+} \times \mathbb{R}^{n}$, we have

$$
\vartheta_{\varepsilon}(t, x) \leq \vartheta(t, x) \quad \forall \varepsilon>0
$$

Let $(t, x)$ be given and let

$$
\delta:=\inf _{y_{x} \in S_{[0, t]}(x)} \max _{\theta \in[0, t]} d\left(y_{x}(\theta), \mathcal{K}\right) .
$$

First we suppose that $\delta>0$. Then for any trajectory $y=y_{x} \in S_{[0, t]}(x)$ there exists some $\theta^{y} \in[0, t]$ such that $d\left(y_{x}\left(\theta^{y}\right), \mathcal{K}\right) \geq \delta$. Hence $\vartheta(t, x)=1$ and $\vartheta_{\varepsilon}(t, x) \geq \inf _{y \in S_{[0, t]}(x)} g_{\varepsilon}\left(y\left(\theta^{y}\right)\right)$, and by $(6.34)$, we get:

$$
\vartheta_{\varepsilon}(t, x) \geq \lim _{\varepsilon \rightarrow 0} \inf _{\{z: d(z, \mathcal{K}) \geq \delta\}} g_{\varepsilon}(z)=1
$$

We conclude that $\lim _{\varepsilon \rightarrow 0} \vartheta_{\varepsilon}(t, x)=1=\vartheta(t, x)$.

Now, we consider the case when $\delta=0$. Assume that there exists some $\eta>0$ such that

$$
\liminf _{\varepsilon \rightarrow 0} \vartheta_{\varepsilon}(t, x) \leq \vartheta(t, x)-\eta
$$

Therefore, there exists a subsequence $\varepsilon_{k}>0$ for $k \geq 1$, and a subsequence $\left(y_{x}^{k}\right)_{k} \subset S_{[0, t]}(x)$ such that

$$
\max \left(\varphi\left(y_{x}^{k}(t)\right), \max _{\theta \in[0, t]} g_{\varepsilon}\left(y_{x}^{k}(\theta)\right)\right) \leq \vartheta(t, x)-\frac{\eta}{2}
$$

In particular,

$$
\max _{\theta \in[0, t]} g_{\varepsilon}\left(y_{x}^{k}(\theta)\right) \leq \vartheta(t, x)-\frac{\eta}{2} \leq 1-\frac{\eta}{2}
$$

Taking into account assumptions $(\mathrm{H} 1)-(\mathrm{H} 5)$, we can extract from $y_{x}^{k}$ a convergent subsequence (in the $L^{\infty}$ norm) to some limit trajectory $y \in S_{[0, t]}(x)$. Now, if there exists $\theta \in[0, t]$ such that $y_{x}(\theta)$ does not belong to $\mathcal{K}$. Then we would obtain $1 \leq 1-\frac{\eta}{2}$. Hence the limit trajectory $y_{x}$ is admissible: $y_{x} \in S_{[0, t]}^{\mathcal{K}}(x)$. Going back to $(6.37)$, and passing to the limit when $\varepsilon \rightarrow 0$, we obtain

$$
\max \left(\varphi\left(y_{x}(t)\right),-M_{0}\right) \leq \vartheta(t, x)-\frac{\eta}{2}
$$

By definition of $\varphi$, this implies that

$$
\varphi\left(y_{x}(t)\right) \leq \vartheta(t, x)-\frac{\eta}{2}
$$

which is a contradiction to the definition of the value function $\varphi$. 


\section{REFERENCES}

[1] J.-P. Aubin and A. Cellina, Differential inclusions, Comprehensive studies in mathematics 264. Springer, Berlin, Heidelberg, New York, Tokyo (1984).

[2] J.-P. Aubin and H. Frankowska, Set-valued analysis, Systems and Control: Foundations and Applications 2. Birkhäuser Boston Inc., Boston (1990).

[3] M. Bardi and I. Capuzzo-Dolcetta, Optimal control and viscosity solutions of Hamilton-Jacobi-Bellman equations, Systems and Control: Foundations and Applications. Birkhäuser, Boston (1997).

[4] M. Bardi, P. Goatin and H. Ishii, A Dirichlet type problem for nonlinear degenerate elliptic equations arising in time-optimal stochastic control. Adv. Math. Sci. Appl. 10 (2000) 329-352.

[5] G. Barles, Solutions de viscosité des équations de Hamilton-Jacobi, Mathématiques et Applications 17. Springer, Paris (1994).

[6] G. Barles and B. Perthame, Discontinuous solutions of deterministic optimal stopping time problems. RAIRO: Modél. Math. Anal. Numér. 21 (1987) 557-579.

[7] G. Barles and B. Perthame, Exit time problems in optimal control and vanishing viscosity method. SIAM J. Control Optim. 26 (1988) 1133-1148

[8] G. Barles and B. Perthame, Comparaison principle for Dirichlet-type Hamilton-Jacobi equations and singular perturbations of degenerated elliptic equations. Appl. Math. Optim. 21 (1990) 21-44.

[9] E.N. Barron, Viscosity solutions and analysis in $L^{\infty}$, in Proceedings of the NATO advanced Study Institute (1999) 1-60.

[10] E.N. Barron and R. Jensen, Semicontinuous viscosity solutions for Hamilton-Jacobi equations with convex Hamiltonians. Commun. Partial Diff. Equ. 15 (1990) 1713-1742.

[11] A. Blanc, Deterministic exit time problems with discontinuous exit cost. SIAM J. Control Optim. 35 (1997) 399-434.

[12] O. Bokanowski, N. Forcadel and H. Zidani, Reachability and minimal times for state constrained nonlinear problems without any controllability assumption. SIAM J. Control Optim. 48 (2010) 4292-4316.

[13] I. Capuzzo-Dolcetta and P.-L. Lions, Hamilton-Jacobi equations with state constraints. Trans. Amer. Math. Soc. 318 (1990) 643-683.

[14] P. Cardaliaguet, M. Quincampoix and P. Saint-Pierre, Optimal times for constrained nonlinear control problems without local controllability. Appl. Math. Optim. 36 (1997) 21-42.

[15] F. Clarke, Y.S. Ledyaev, R. Stern and P. Wolenski, Nonsmooth analysis and control theory. Springer (1998).

[16] H. Frankowska, Lower semicontinuous solutions of Hamilton-Jacobi-Bellman equations. SIAM J. Control Optim. 31 (1993) $257-272$.

[17] H. Frankowska and S. Plaskacz, Semicontinuous solutions of Hamilton-Jacobi-Bellman equations with degenerate state constraints. J. Math. Anal. Appl. 251 (2000) 818-838.

[18] H. Frankowska and R.B. Vinter, Existence of neighboring feasible trajectories: applications to dynamic programming for state-constrained optimal control problems. J. Optim. Theory Appl. 104 (2000) 21-40.

[19] H. Ishii and S. Koike, A new formulation of state constraint problems for first-order PDEs. SIAM J. Control Optim. 34 (1996) $554-571$.

[20] M. Motta, On nonlinear optimal control problems with state constraints. SIAM J. Control Optim. 33 (1995) $1411-1424$.

[21] H.M. Soner, Optimal control with state-space constraint, I. SIAM J. Control Optim. 24 (1986) 552-561.

[22] H.M. Soner, Optimal control with state-space constraint, II. SIAM J. Control Optim. 24 (1986) 1110-1122.

[23] P. Soravia, Optimality principles and representation formulas for viscosity solutions of Hamilton-Jacobi equations. II. Equations of control problems with state constraints. Diff. Int. Equ. 12 (1999) 275-293. 